PACIFIC JOURNAL OF MATHEMATICS

Vol. 177, No. 2, 1997

\title{
ON FUSION ALGEBRAS ASSOCIATED TO FINITE GROUP ACTIONS
}

\author{
Hideki Kosaki, Akihiro Munemasa and Shigeru Yamagami
}

In our previous paper (see Kosaki and Yamagami), four kinds of bimodules naturally attached to crossed products $P \rtimes G \supseteq P \rtimes H$ determined by a group-subgroup pair $G \supseteq H$ were identified with certain vector bundles equipped with group actions. In the present paper we will describe the structure of the fusion algebra of vector bundles and clarify a relationship to fusion algebras appearing in other contexts. Some applications to automorphism analysis for subfactors will be also given.

\section{Introduction.}

The notion of bimodules (for von Neumann algebras) was introduced by A. Connes and has proved very useful in many places in the theory of operator algebras. The Jones index theory (and subsequent subfactor analysis) is no exception. A given factor-subfactor pair $M \supseteq N$ (of finite index) naturally provides us with four kinds of bimodules (i.e., $M-M, M-N, N-M, N-N$ bimodules) via the basic construction. These bimodules are known to contain enormous amount of information on the pair $M \supseteq N$, and hence study on bimodules is indispensable for subfactor analysis. For example, knowing how tensor products split into irreducible bimodules (i.e., fusion rule) is very useful. An important example of factor-subfactor pairs is the crossed product pair $M=P \rtimes G \supseteq N=P \rtimes H$ arising from a group-subgroup pair $G \supseteq H$. In this case, the four kinds of bimodules and the inductionrestriction procedure for them (i.e., the principal and the dual principal graphs $([8]))$ can be explicitly described in terms of the representation theory for the pair $G \supseteq H$ (i.e., the Mackey machine). Actually, in our previous paper [22], via a certain crossed product construction a tensor category of vector bundles having groups as base spaces with bivariant actions of subgroups was introduced. We then showed that (based on the outerness of an action in question) this can be identifed with the category of the above mentioned bimodules, and hence study of bimodules was completely reduced to that in representation theory ( see $\S 2$ ). 
The fusion rule for $M-M$ bimodules is quite easy in the group-subgroup case, and the purpose of the present paper is to determine much more subtle fusion rule for $N-N$ bimodules together with some applications to the study on automorphisms for subfactors. It turns out that the fusion algebra for $N-N$ bimodules is essentially the same as the algebra of Hecke operators considered by T. Yoshida $([40,41])$. We give a concrete description of the fusion algebra based on [28], revealing the abstract structure as a semisimple algebra. Motivated mainly by Verlinde's work ([34]), fusion algebras in several contexts have been investigated so far. Among these, the fusion algebra of the orbifold model appearing in [5] (see also [1, 2, 27, 35]) with the trivial 3-cocycle is a special case of our fusion algebra of vector bundles, where $G=H \times H$ with the diagonally imbedded $H$.

In $\S 3$ we obtain a multiplicity formula for an irreducible bundle in the tensor product of bundles in quite a general setting. In $\S 4$ we study the fusion algebra of vector bundles and obtain a concrete realization of this algebra. We also point out that the fusion algebra of the asymptotic inclusion (see [30]) of $\mathcal{R}_{0} \rtimes G \supseteq \mathcal{R}_{0}$ (where $\mathcal{R}_{0}$ is the hyperfinite $\mathrm{II}_{1}$ factor) is the center of the quantum double $([\mathbf{6}])$ of the group Hopf algebra $\ell^{\infty}(G)$. This fact for the fusion algebra arising from an orbifold model $([\mathbf{1}, \mathbf{2}, \mathbf{5}])$ is probably known to several people, especially to M. Wakui ([35], see also [4]). Then, in $\S 5$ we identify elements in the fusion algebra with operators acting on a certain character ring (considered in $[\mathbf{4 0}, \mathbf{4 1}]$ ). This enables us to express the tensor product very explicitly in terms of relevant representations. In subfactor analysis, certain groups of automorphisms with special properties naturally appear (i.e., for example the group of non-strongly outer automorphisms $([3,19,32]))$ and they are known to be related to analysis on bimodules. In the final $\S 6$, we explicitly write down these groups for an inclusion of fixed-point algebra factors.

Throughout the paper we follow the notations and definitions in our previous paper [22] (which are summarized in $\S 2$ ) while basic facts on the index theory can be found in the original articles $[8, \mathbf{1 4}, \mathbf{1 8}]$. The authors are grateful to Y. Kawahigashi for informing them of $[7,16]$ in preparation.

\section{Preliminaries.}

In this section we briefly recall basic definitions and results in [22] partly to fix our notations.

2.1. Vector Bundles. Let $\Gamma$ be a discrete group, and its finite subgroups will be denoted by $G, H, K, \cdots$. The unit in the group $\Gamma$ will be denoted by 1 throughout the paper. By an $H$ - $K$ vector bundle $V\left(={ }_{H} V_{K}\right)$ we always mean a vector bundle of Hilbert spaces over the base space $\Gamma$ with left $H$ - 
and right $K$-actions which extend the natural left $H$ - and right $K$-actions on the base space and preserve inner products (in fibre Hilbert spaces). We allow some of fibres $V_{g}$ to be trivial, and $\mathbf{s}(V)=\left\{g \in \Gamma ; V_{g} \neq\{0\}\right\}$ is called the support of $V$. The direct sum and the adjoint bundle are defined in the natural way $\left(V^{*}={ }_{K}\left(V^{*}\right)_{H}\right.$ with $\left(V^{*}\right)_{g}=\left(V_{g^{-1}}\right)^{*}$, the dual space) while the tensor product is defined as follows: Let $V, W$ be $G-H, H-K$ bundles respectively. The outer tensor product $V \otimes W$ over $\Gamma \times \Gamma$ admits the $H$-action

$$
h \cdot(v \otimes w)=\left(v h^{-1}\right) \otimes(h v) .
$$

Hence, we get the quotient bundle $V \otimes_{H} W$ over the quotient space $\Gamma \times_{H} \Gamma$ of $\Gamma \times \Gamma$. The product bundle $V \otimes_{H} W={ }_{G}\left(V \otimes_{H} W\right)_{K}$ is defined as the pushforward of $V \bigotimes_{H} W$ via the map $p: g \times_{H} g^{\prime} \in \Gamma \times_{H} \Gamma \longrightarrow g g^{\prime} \in \Gamma$. Hence, we get

$$
\left(V \otimes_{H} W\right)_{g}=\bigoplus_{a \times_{H} b \in p^{-1}(g)}\left(V_{a} \otimes_{H} W_{b}\right),
$$

and we have the natural $G-K$ action from the outside.

For $H$ - $K$ bundles $V, W$, a bundle homomorphism $T: V \longrightarrow W$ satisfying $T\left(V_{g}\right) \subseteq W_{g}$ for each $g \in \Gamma$ is called an $(H-K)$ gauge transformation, and the set of all gauge transformations is denoted by $\operatorname{Hom}(V, W)$ (and $\operatorname{Hom}(V, V)=$ $\operatorname{Hom}(V)$ as usual). A bundle $V$ is irreducibe when it does not contain a nontrivial $H-K$ subbundle. The irreducibility obviously forces that the support $\mathbf{s}(V)$ consists of a single $H$ - $K$ orbit, say $O$. For each $g \in O$ and $T \in \operatorname{Hom}(V)$, $t=\left.T\right|_{V_{g}}$ satisfies

$$
t(h v k)=h t(v) k \text { for all } v \in V_{g} \text { and }(h, k) \in H \times K \text { with } h g k=g .
$$

Denoting all such $t$ 's by $\operatorname{Hom}\left(V_{g}\right)$, we observe that the irreducibility of $V$ means $\operatorname{Hom}\left(V_{g}\right)=\mathbb{C} 1$. We set $H \times{ }_{g} K=\{(h, k) \in H \times K ; h g=g k\}$ (the stabilizer at $g$ under the action $\left.(h, k) \cdot g=h g k^{-1}\right)$, which is isomorphic to $H \cap g K g^{-1}$ and $g^{-1} H g \cap K$ via

$$
H \times{ }_{g} K=\left\{\left(h, g^{-1} h g\right) ; h \in H \cap g K g^{-1}\right\}=\left\{\left(g k g^{-1}, k\right) ; k \in g^{-1} H g \cap K\right\} .
$$

The above requirement for $t$ is nothing but the intertwining property for the representation $(h, k) \cdot v=h v k^{-1}$ of $H \times{ }_{g} K$ (determined by $V$, or more precisely by $V_{g}$ ). Hence, the irreducibility of $V$ is equivalent to that of the above representation. The dependency coming from a choice of $g \in O$ is redundant, and hence each irreducible vector bundle is parameterized by an orbit and an irreducible representation of the relevant stabilizer.

The irreducible bundle ${ }_{H} \mathbb{C}_{K}$ (called the trivial bundle) corresponding to the orbit $H K=H 1 K$ and the identity representation of $H \times{ }_{1} K \cong H \cap K$ serves as a generating object in our analysis (see 2.4). 
2.2. Bimodules. Let $N, M$ be factors. An $N-M$ bimodule $\mathcal{K}={ }_{N} \mathcal{K}_{M}$ is a Hilbert space equipped with commuting normal actions of $N$ and the opposed algebra of $M$. (As usual, we regard the action of the opposed algebra of $M$ as a right $M$-action.) The direct sums and contragredient bimodules are defined in the usual way. For example, the contragredient bimodule $\mathcal{K}^{*}={ }_{M}\left(\mathcal{K}^{*}\right)_{N}$ is the dual space, that is, the Hilbert space of antivectors $\bar{\xi}(\xi \in \mathcal{K})$ equipped with the action $m \cdot \bar{\xi} \cdot n=\overline{n^{*} \cdot \xi \cdot m^{*}}$. The notion of a relative tensor product $\mathcal{K} \otimes_{N} \mathcal{L}$ (which is an $M$ - $P$ bimodule) of an $M$ $N$ bimodule $\mathcal{K}$ and an $N-P$ bimodule $\mathcal{L}$ was studied in [33]. The identity bimodule ${ }_{M} L^{2}(M)_{M}$ is the standard Hilbert space $L^{2}(M)$ with the action $m_{1} \cdot \xi \cdot m_{2}=m_{1} J_{M} m_{2}^{*} J_{M} \xi$, and it is the multiplicative unit for the relative tensor product. (Here, $J_{M}$ denotes the modular conjugation.)

For an $N-M$ bimodule $\mathcal{K}$, the index of $\mathcal{K}$ means the the minimal index $([\mathbf{1 0}, \mathbf{1 1}, \mathbf{2 4}]$, see also $[\mathbf{2 0}, \mathbf{2 5}])$ of $N$ sitting in the commutant of the right $M$-action. (Notice that the relative commutant between these two factors is the algebra of self-intertwiners.) The square root of the index is called the dimension of $\mathcal{K}$. In this paper we will exclusively deal with bimodules with finite index. Hence, a bimodule (of finite index) can be always decomposed into the sum of (finitely many) irreducible bimodules in a canonical way by choosing minimal projections in the (finite dimensional) algebra of selfintertwiners. The importance of the notion of dimension lies in the fact that it is additive and multiplicative for direct sums and relative tensor products respectively. Details on these facts can be found in $[31,36,37]$.

When $N \subseteq M$ is a factor-subfactor pair with finite index ([14]), the $N$ $M$ bimodule $\mathcal{H}=L^{2}(M)$ with the action $n \cdot \xi \cdot m=n J_{M} m^{*} J_{M} \xi$ plays an important role in subfactor analysis (see $[\mathbf{2 9}, \mathbf{3 0}]$ and 2.4).

2.3. Categorical equivalence. Let $\alpha: \Gamma \longrightarrow \operatorname{Aut}(P)$ be an outer action on a factor $P$ so that $A=P \rtimes_{\alpha} H$ and $B=P \rtimes_{\alpha} K$ are factors. From a given $H$ - $K$ bundle $V$, we try to construct an $A$ - $B$ bimodule by mimicking the construction of a crossed product. Let $\hat{V}$ be the Hilbert space $\oplus_{g \in \Gamma}\left(L^{2}(P) \otimes\right.$ $V_{g}$ ). We let $P$ act on $\hat{V}$ from the left and $K$ (identified with $\lambda_{k}$ 's in the crossed product) act on $\hat{V}$ from the right in the obvious way. The right $P$-action and the left $H$-action are defined by

$$
\begin{aligned}
\left(\sum_{g} \xi_{g} \otimes v_{g}\right) \cdot p & =\sum_{g} \xi_{g} \alpha_{g}(p) \otimes v_{g}, \\
h \cdot\left(\sum_{g} \xi_{g} \otimes v_{g}\right) & =\sum_{g} u_{h} \xi_{g} \otimes h v_{g},
\end{aligned}
$$

where $u_{h}$ is the canonical implementation of $\alpha_{h}$. Then, $\hat{V}$ becomes an $A$ - $B$ bimodule. We know that the correspondence $V \longrightarrow \hat{V}$ gives an equivalence 
of categories of vector bundles and bimodules (Theorem 1.7, [22], see also $[38])$.

2.4. Principal and dual principal graphs. Let $H \subseteq G=\Gamma$ in 2.1 and 2.3 and we set $V={ }_{H} \mathbb{C}_{G}$. Then, the decomposition rules for

$$
\begin{aligned}
& { }_{H} \mathbb{C}_{H}, V, V \otimes_{G} V^{*}, V \otimes_{G} V^{*} \otimes_{H} V, \cdots(H-H \text { and } H-G \text { bundles }) \\
& { }_{G} \mathbb{C}_{G}, V^{*}, V^{*} \otimes_{H} V, V^{*} \otimes_{H} V \otimes_{G} V^{*}, \cdots(G-G \text { and } G-H \text { bundles })
\end{aligned}
$$

describe the principal and the dual principal graphs for the inclusion $M=$ $P \rtimes_{\alpha} G \supseteq N=P \rtimes_{\alpha} H$. In fact, since $V$ corresponds to $\mathcal{H}$ in 2.2 , the above two sequences correspond to the basic sequences of bimodules (which give us the four kinds of bimodules, the principal graph and the dual principal graph) in Ocneanu's approach $([29,30])$. As usual we may and do assume $\left\{h \in H ; g h g^{-1} \in H\right.$ for all $\left.g \in G\right\}=\{1\}$. Then, the irreducible $G-G, G-H$ (or $H-G$ ) bundles appearing in the above two sequences (i.e., $M-M, M-N$ (or $N-M$ ) bimodules) are parameterized by the irreducible representations $\hat{G}, \hat{H}$ respectively. (Notice that we have only one $G-H$ orbit $G=G 1 H$ for example.) On the other hand, a description of the irreducible $H-H$ bundles appearing in the first sequence (i.e., $N-N$ bimodules) is more complicated. Let $\left\{H g_{i} H\right\}_{i=1,2, \cdots, n}$ be the $H-H$ orbits in $\Gamma=G$ (i.e., $g_{i}$ 's form a complete set of representatives for $H \backslash G / H)$ with $g_{1}=1$, and we set $H_{i}=g_{i} H g_{i}^{-1} \cap H\left(\cong H \times g_{i} H\right.$, the stabilizer $)$. Then the irreducible $H-H$ bundles are parameterized by the disjoint union $\bigsqcup_{i} \hat{H}_{i}$.

Tensoring with $V$ or $V^{*}$ (from suitable directions) corresponds to either restriction or induction. Therefore, as was shown in $\S 3,[22]$, the dual principal graph of $M \supseteq N$ is given by

$$
\text { ind }: \alpha \rightarrow \operatorname{Ind}_{H}^{G} \alpha \text { and rest }: \beta \rightarrow \operatorname{Rest}_{H}^{G} \beta
$$

between $\hat{H}$ and $\hat{G}$ while the more subtle principal graph is given by

$$
\text { ind }: \gamma \in \hat{H}_{i} \rightarrow \operatorname{Ind}_{H_{i}}^{H} \gamma \text { and rest }: \delta \in \hat{H} \rightarrow \bigoplus_{i} \operatorname{Rest}_{H_{i}}^{H} \delta
$$

between $\hat{H}$ and $\bigsqcup_{i} \hat{H}_{i}$.

\section{Multiplicity Formula.}

In this section, we shall establish a multiplicity formula for the decomposition of tensor products of irreducible vector bundles. By the Frobenius reciprocity ([39]), it suffices to know the multiplicity of the trivial bundle in triple tensor products. 
Let $G, H, K$ be finite subgroups of a discrete group $\Gamma$ as in 2.1, and let $U$, $V$, and $W$ be irreducible $G-H, H-K$, and $K-G$ bundles with supports $\mathbf{s}(U)$, $\mathbf{s}(V)$, and $\mathbf{s}(W)$ respectively. Then we need to describe the dimension of the space $\operatorname{Hom}\left({ }_{G} \mathbb{C}_{G},{ }_{G} U \otimes_{H} V \otimes_{K} W_{G}\right)$ of intertwiners. To this end, we first derive an expression for the relative tensor product bundle $U \otimes_{H} V \otimes_{K} W$ in terms of a vector bundle defined on the set

$\Omega^{\prime}=\left\{(a, b, c, d) \in \Gamma \times \Gamma \times \Gamma \times \Gamma ; a b^{-1} \in \mathbf{s}(U), b c^{-1} \in \mathbf{s}(V), c d^{-1} \in \mathbf{s}(W)\right\}$

Clearly $\Omega^{\prime}$ is invariant under the left multiplication of $G \times H \times K \times G$ and the diagonal translation of $\Gamma$ from right. Furthermore,

$$
(a, b, c, d) \in \Omega^{\prime} \mapsto\left(a b^{-1}, b c^{-1}, c d^{-1}\right) \in \mathbf{s}(U) \times \mathbf{s}(V) \times \mathbf{s}(W)
$$

defines a $G \times H \times K \times G$-equivariant surjection. It is immediate to check that each fibres consist of (diagonal) $\Gamma$-orbits and hence this map gives rise to the identification $\Omega^{\prime} / \Gamma \cong \mathbf{s}(U) \times \mathbf{s}(V) \times \mathbf{s}(W)$. Then the outer tensor product bundle $U \otimes V \otimes W$ is regarded as a vector bundle over $\Omega^{\prime} / \Gamma$ and, dividing by the $H \times K$ action, we obtain the identification:

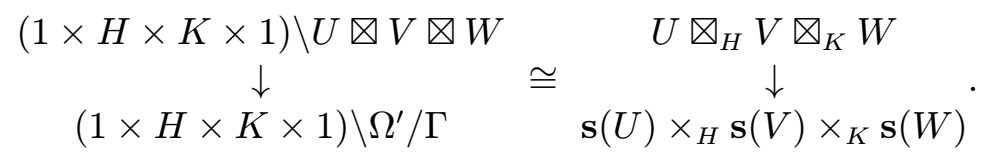

$\left(U \otimes_{H} V\right.$ refers to the quotient of $U \otimes V$ under the left action of $H$ defined by $h \cdot(u \otimes v)=u h^{-1} \otimes h v$ and similarly for $V \bigotimes_{K} W$ (see 2.1).) Note that this identification intertwines $G \times 1 \times 1 \times G$ and $G$ - $G$ actions.

Let $E^{\prime}$ be the pullback of $U \otimes V \otimes W$ under the map $\Omega^{\prime} \rightarrow \Omega^{\prime} / \Gamma$. Then the construction just explained is compactly expressed by

$$
U \otimes_{H} V \otimes_{K} W=(1 \times H \times K \times 1) \backslash E^{\prime} / \Gamma .
$$

Let $p:(1 \times H \times K \times 1) \backslash \Omega^{\prime} / \Gamma \rightarrow \Gamma$ be the map defined by

$$
p([a, H b, K c, d])=a d^{-1}
$$

(the bracket means the quotient by the diagonal $\Gamma$-action).

By the definition of relative tensor product bundles, we see that $U \otimes_{H} V \otimes_{K}$ $W$ is isomorphic to the pushforward (or the fibre direct sum) of $U \otimes_{H} V \otimes_{K} W$ with respect to $p$ and hence we have to describe the vector space

$$
\operatorname{Hom}\left({ }_{G} \mathbb{C}_{G},{ }_{G} p_{*}\left(U \otimes_{H} V \bigotimes_{K} W\right)_{G}\right) .
$$


Since ${ }_{G} \mathbb{C}_{G}$ denotes the trivial $G$ - $G$ bundle, the following lemma shows that this space is identified with the vector space of invariant vectors under the adjoint action of $G$

$$
\left(\bigoplus_{[a, H b, K c, d] \in p^{-1}(1)} U_{a b^{-1}} \bigotimes_{H} V_{b c^{-1}} \otimes_{K} W_{c d^{-1}}\right)^{\operatorname{Ad} G} .
$$

Lemma 1. Let $G$ be a finite group and ${ }_{G} X,{ }_{G} Y$ be $G$-spaces. Let $E$ be a G-vector bundle over $X$ and $p: X \rightarrow Y$ be a G-equivariant map. Take an element $y \in Y$ in the image of $p$. Then we have the following natural isomorphism:

$$
\operatorname{Hom}\left(G y \times \mathbb{C},\left.p_{*}(E)\right|_{G y}\right) \cong\left(\bigoplus_{x \in p^{-1}(y)} E_{x}\right)^{G(y)} .
$$

Here, Hom in the left hand side refers to the vector space of G-linear bundle maps which make the base space point-wise invariant while the right hand side denotes the space of vectors invariant under the isotropy subgroup $G(y)=\{g \in G ; g y=y\}$.

Since the fibre $p^{-1}(1)$ is given by $\left\{[a, H b, K c, a] ;(a, b, c, a) \in \Omega^{\prime}\right\}$, regarding

$$
\Omega \equiv\left\{(a, b, c) \in \Gamma \times \Gamma \times \Gamma ; a b^{-1} \in \mathbf{s}(U), b c^{-1} \in \mathbf{s}(V), c a^{-1} \in \mathbf{s}(W)\right\}
$$

as the set $\left\{(a, b, c, a) \in \Omega^{\prime}\right\}$ and letting $E=\left.E^{\prime}\right|_{\Omega}$, we see that the vector space $\left.\left(U \otimes_{H} V \bigotimes_{K} W\right)\right|_{p^{-1}(1)}$ is identified with

$$
(1 \times H \times K) \backslash E / \Gamma \text {. }
$$

Since the adjoint action of $G$ on $\left.\left(U \otimes_{H} V \bigotimes_{K} W\right)\right|_{p^{-1}(1)}$ is transfered into the left multiplication of $G \times 1 \times 1$ on $E \rightarrow \Omega$, we have proved the following:

\section{Lemma 2.}

$$
\operatorname{Hom}\left({ }_{G} \mathbb{C}_{G},{ }_{G} U \otimes_{H} V \otimes_{K} W_{G}\right) \cong(G \times H \times K) \backslash \hat{E} / \Gamma .
$$

Here, $E=\left\{E_{(a, b, c)}=U_{a b^{-1}} \otimes V_{b c^{-1}} \otimes W_{c a^{-1}}\right\}_{(a, b, c) \in \Omega}$ is the vector bundle over $\Omega$ with the $(G \times H \times K)-\Gamma$ action defined by

$$
(g, h, k)(a, b, c ; u \otimes v \otimes w) \gamma=\left(g a \gamma, h b \gamma, k c \gamma ; g u h^{-1} \otimes h v k^{-1} \otimes k w g^{-1}\right),
$$

and $\hat{E}$ is the vector space $\oplus_{\omega \in \Omega} E_{\omega}$ with the obvious $(G \times H \times K)-\Gamma$ action.

Theorem 3. The multiplicity of the trivial bundle in $U \otimes_{H} V \otimes_{K} W$ is given by

$$
\sum_{1 \leq l \leq d}\left|\Gamma\left(G a_{l}, H b_{l}, K c_{l}\right)\right|^{-1} \sum_{\gamma \in \Gamma\left(G a_{l}, H b_{l}, K c_{l}\right)} \xi_{l}(\gamma) \eta_{l}(\gamma) \zeta_{l}(\gamma)
$$


Here, $\left\{\left(G a_{l}, H b_{l}, K c_{l}\right)\right\}_{1 \leq l \leq d}$ denotes a set of representatives of $\Gamma$-orbits in $(G \times H \times K) \backslash \Omega, \Gamma\left(G a_{l}, H b_{l}, K c_{l}\right)=\left(a_{l}^{-1} G a_{l}\right) \cap\left(b_{l}^{-1} H b_{l}\right) \cap\left(c_{l}^{-1} K c_{l}\right)$ and $\xi_{l}$, $\eta_{l}, \zeta_{l}$ refer to the characters of $U, V, W$ at $a_{l} b_{l}^{-1}, b_{l} c_{l}^{-1}, c_{l} a_{l}^{-1}$ respectively. (For example, $\xi_{l}$ is the character of the representation of $a_{l}^{-1} G a_{l} \cap b_{l}^{-1} H b_{l}$ at $U_{a_{l} b_{l}^{-1}}$ defined by $u \in U_{a_{l} b_{l}^{-1}} \mapsto\left(a_{l} \gamma a_{l}^{-1}\right) u\left(b_{l} \gamma b_{l}^{-1}\right)^{-1} \in U_{a_{l} b_{l}^{-1}}$ for $\gamma \in$ $a_{l}^{-1} G a_{l} \cap b_{l}^{-1} H b_{l}$.)

Proof. First recall that $E_{(a, b, c)}=U_{a b^{-1}} \otimes V_{b c^{-1}} \otimes W_{c a^{-1}}$ and the fibre of the quotient vector bundle $(G \times H \times K) \backslash E$ at $(G a, H b, K c) \in(G \times H \times K) \backslash \Omega$ is identified with the set of $G \times H \times K$-equivariant sections of $E$ over $G a \times$ $H b \times K c$.

Since the action of $G \times H \times K$ on $\Omega$ is free, we need to calculate the dimension of the vector space of $\Gamma$-equivariant sections of $(G \times H \times K) \backslash E$ on each $\Gamma$-orbits in $(G \times H \times K) \backslash \Omega$. For $(G a, H b, K c) \in(G \times H \times K) \backslash \Omega$, denote by

$$
\begin{aligned}
\Gamma(G a, H b, K c) & =\{\gamma \in \Gamma ;(G a \gamma, H b \gamma, K c \gamma)=(G a, H b, K c)\} \\
& =\left(a^{-1} G a\right) \cap\left(b^{-1} H b\right) \cap\left(c^{-1} K c\right)
\end{aligned}
$$

the stabilizer of the right action of $\Gamma$ at $(G a, H b, K c)$. Let $\gamma \in \Gamma(G a, H b, K c)$ and define $g \in G, h \in H, k \in K$ by

$$
a \gamma=g a, b \gamma=h b, c \gamma=k c .
$$

Then the action of $\gamma$ on $((G \times H \times K) \backslash E)_{(G a, H b, K c)}$ is given by left multiplication of $(g, h, k)$, i.e.,

$$
\begin{aligned}
& \xi \otimes \eta \otimes \zeta \in U_{a b^{-1}} \otimes V_{b c^{-1}} \otimes W_{c a^{-1}} \\
& \mapsto g \xi h^{-1} \otimes h \eta k^{-1} \otimes k \zeta g^{-1} \in U_{a b^{-1}} \otimes V_{b c^{-1}} \otimes W_{c a^{-1}},
\end{aligned}
$$

which shows that the (right) action of $\gamma \in \Gamma(G a, H b, K c)$ on $((G \times H \times$ $K) \backslash E)_{(G a, H b, K c)}$ is identified with the (left) action of

$$
\begin{aligned}
\left(a \gamma a^{-1}, b \gamma b^{-1}\right) \times\left(b \gamma b^{-1}, c \gamma c^{-1}\right) \times & \left(c \gamma c^{-1}, a \gamma a^{-1}\right) \in\left(G \times_{a b^{-1}} H\right) \times \\
& \left(H \times_{b c^{-1}} K\right) \times\left(H \times_{c a^{-1}} G\right)
\end{aligned}
$$

on $U_{a b^{-1}} \otimes V_{b c^{-1}} \otimes W_{c a^{-1}}$.

Thus, the value of the character of $\gamma$ on $((G \times H \times K) \backslash E)_{(G a, H b, K c)}$ is given by $\xi_{l}(\gamma) \eta_{l}(\gamma) \zeta_{l}(\gamma)$ and hence the dimension of the fixed point vectors is calculated by

$$
\left|\Gamma\left(G a_{l}, H b_{l}, K c_{l}\right)\right|^{-1} \sum_{\gamma \in \Gamma\left(G a_{l}, H b_{l}, K c_{l}\right)} \xi_{l}(\gamma) \eta_{l}(\gamma) \zeta_{l}(\gamma) .
$$


Remark. A similar argument shows the following: Let $V_{i}, i=1,2, \cdots, N$ be $H$ - $H$ bundles over $\Gamma$. Then $V_{1} \otimes V_{2} \otimes \cdots \otimes V_{N}$ contains the trivial bundle ${ }_{H} \mathbb{C}_{H}$ with multiplicity

$$
\sum_{\alpha}\left|\Gamma\left(H g_{1}^{\alpha}, \cdots, H g_{N}^{\alpha}\right)\right|^{-1} \sum_{g \in \Gamma\left(H g_{1}^{\alpha}, \cdots, H g_{N}^{\alpha}\right)} \chi_{1}^{\alpha}(g) \cdots \chi_{N}^{\alpha}(g),
$$

where $\left\{\left(H g_{1}^{\alpha}, \cdots, H g_{N}^{\alpha}\right)\right\}_{\alpha}$ denotes a set of representatives of the right diagonal $\Gamma$-orbits in $H \backslash \Gamma \times \cdots \times H \backslash \Gamma$ and $\chi_{i}^{\alpha}$ refers to the character of $V_{i}$ at $g_{i}^{\alpha} \in \Gamma$.

\section{Fusion Algebras.}

We keep the notations in the previous sections.

Definition. The fusion algebra $\mathcal{F}$ of bivariant vector bundles over $\Gamma$ is by definition a family $\left\{{ }_{H} \mathcal{F}_{K}\right\}_{H, K}$ of complex vector spaces $(H, K$ are finite subgroups of $\Gamma$ ) universally generated by the set of symbols $\langle V\rangle$ ( $V$ runs through $H-K$ bundles) with the relation

$$
\begin{aligned}
\langle V\rangle & =\langle W\rangle \quad \text { if } \quad V \cong W \\
\langle V \oplus W\rangle & =\langle V\rangle+\langle W\rangle .
\end{aligned}
$$

On $\mathcal{F}$, the ${ }^{*}$-operation and the restricted multiplication (the multiplication can be performed only when subgroup-labelings coincide at the inner side) is defined by

$$
\begin{aligned}
\langle V\rangle^{*} & =\left\langle V^{*}\right\rangle \\
\langle V\rangle\langle W\rangle & =\left\langle V \otimes_{H} W\right\rangle,
\end{aligned}
$$

which furnish $\mathcal{F}$ with a ${ }^{*}$-algebra-like structure.

Note that any element in ${ }_{H} \mathcal{F}_{K}$ is a linear combination of irreducible $\langle V\rangle$ 's.

In the remaining of this section, we exclusively deal with the situation $G=H=K$. Then the fusion algebra ${ }_{H} \mathcal{F}_{H}$ is a usual (unrestricted) * algebra, and here a concrete realization of this algebra will be investigated.

Let $X=H \backslash \Gamma$ be the right coset space and denote by $X(\gamma)$ the set of fixed-points in $X$ under the right translation of $\gamma \in \Gamma$. On the multi-matrix *-algebra $\bigoplus_{\gamma \in \Gamma} \mathcal{B}\left(\ell^{2}(X(\gamma))\right)$, we define a ${ }^{*}$-preserving automorphic action of $\Gamma$ by

$$
(x \gamma)\left(\gamma^{\prime}\right)=R_{\gamma}^{-1} x\left(\gamma \gamma^{\prime} \gamma^{-1}\right) R_{\gamma} \in \mathcal{B}\left(\ell^{2}\left(X\left(\gamma^{\prime}\right)\right)\right) .
$$

Here, $x \in \bigoplus_{\gamma \in \Gamma} \mathcal{B}\left(\ell^{2}(X(\gamma))\right)$ is identified with an operator-valued function on $\Gamma$ and $R_{\gamma}: \ell^{2}\left(X\left(\gamma^{-1} \gamma^{\prime} \gamma\right)\right) \rightarrow \ell^{2}\left(X\left(\gamma^{\prime}\right)\right)$ denotes the unitary map induced 
from the right multiplication of $\gamma$ on $H \backslash \Gamma$. A *-algebra $\mathcal{A}$ is then defined to be the fixed-point algebra under this action:

$$
\mathcal{A}=\left\{x \in \bigoplus_{\gamma \in \Gamma} \mathcal{B}\left(\ell^{2}(X(\gamma))\right) ; x \gamma^{\prime}=x \text { for all } \gamma^{\prime} \in \Gamma\right\}
$$

Note that, if $\left\{\gamma_{\alpha}\right\}$ is a representative set for conjugacy classes of $\Gamma$, then $\mathcal{A}$ is isomorphic to

$$
\bigoplus_{\alpha} \mathcal{A}_{\alpha}
$$

Here, $\mathcal{A}_{\alpha}$ denotes the set of linear operators in $\mathcal{B}\left(\ell^{2}\left(X\left(\gamma_{\alpha}\right)\right)\right)$ which commute with right translations by elements in the centralizer $\left\{\gamma \in \Gamma ; \gamma \gamma_{\alpha}=\gamma_{\alpha} \gamma\right\}$ of $\gamma_{\alpha}$.

We now associate an element $x_{V}$ in $\mathcal{A}$ to each $H$ - $H$ bundle $V$ over $\Gamma$. To describe this, we use the expression of $V$ as a vector bundle over $\Gamma \times \Gamma$ : Let $\pi:\left(\gamma_{1}, \gamma_{2}\right) \in \Gamma \times \Gamma \mapsto \gamma_{1} \gamma_{2}^{-1} \in \Gamma$ and let $\pi^{*} V$ be the pullback bundle of $V$ by $\pi$. As in the previous part, we consider $\Gamma \times \Gamma$ to be a left $H \times H$-and right $\Gamma$ - space. Then $\pi^{*} V$ admits a natural $H \times H$ - $\Gamma$ action which extends the $H \times H-\Gamma$ action on the base space $\Gamma \times \Gamma$. Since fibres of $\pi$ are given by $\Gamma$-orbits and since $\Gamma$ acts on $\Gamma \times \Gamma$ freely, to consider an $H$ - $H$ bundle over $\Gamma$ is equivalent to consider an $(H \times H)$ - $\Gamma$ bundle over $\Gamma \times \Gamma$ (take pullbacks by $\pi$ or quotients by $\Gamma$-action).

Since the (left) $H \times H$-action on $\Gamma \times \Gamma$ is free as well, we can first take the quotient of $\pi^{*} V$ by $H \times H$-action. Then the equivariance condition is coded into the diagonal $\Gamma$-action on the vector bundle $(H \times H) \backslash \pi^{*} V$ over $X \times X=(H \backslash \Gamma) \times(H \backslash \Gamma)$.

In particular, if we denote by $\Gamma(x, y)$ the isotropy subgroup of $\Gamma$ at $(x, y) \in$ $X \times X$, then each $\gamma \in \Gamma(x, y)$ gives rise to a linear operator in the fibre $\left(H \times H \backslash \pi^{*} V\right)_{(x, y)}$. Denote by $\chi_{V}^{(x, y)}(\gamma)$ the trace of that operator (i.e., the character of $\Gamma(x, y)$ and we set $\chi_{V}^{(x, y)}=0$ if $\left.\left(H \times H \backslash \pi^{*} V\right)_{(x, y)}=0\right)$ and define the element $x_{V}$ in $\mathcal{A}$ by

$$
x_{V}=\sum_{x, y \in X} \sum_{\gamma \in \Gamma(x, y)} \chi_{V}^{(x, y)}(\gamma)(x, y ; \gamma)
$$

where $(x, y ; \gamma)$ is the linear operator in $\mathcal{B}\left(\ell^{2}(X(\gamma))\right)$ defined by

$$
((x, y ; \gamma) f)(z)=\delta_{x, z} f(y)
$$

i.e., $\{(x, y ; \gamma)\}_{x, y \in X(\gamma)}$ forms a matrix unit in $\mathcal{B}\left(\ell^{2}(X(\gamma))\right)$ with respect to the orthogonal basis of point-evaluation functions on $X(\gamma)$. 
Theorem 4. Let $\Gamma$ be a finite group, $H$ a subgroup of $\Gamma$. Then the correspondence $\langle V\rangle \in{ }_{H} \mathcal{F}_{H} \mapsto x_{V} \in \mathcal{A}$ is well-defined and gives rise to a ${ }^{*}$ isomorphism.

Proof. Equip $\oplus_{\gamma \in \Gamma} \mathcal{B}\left(\ell^{2}(X(\gamma))\right)$ with an inner product for which the matrix units $\{(x, y ; \gamma) ; x, y \in X(\gamma), \gamma \in \Gamma\}$ form an orthogonal basis:

$$
\left\langle(x, y ; \gamma) \mid\left(z, w ; \gamma^{\prime}\right)\right\rangle=\delta_{x z} \delta_{y w} \delta_{\gamma \gamma^{\prime}} \frac{1}{|\Gamma|} .
$$

Then, the set $\left\{x_{V}\right\}$, where $V$ runs through all irreducible $H-H$ bundles on $\Gamma$, forms an orthonormal basis of $\mathcal{A}$. If we denote by $N_{U V}^{W}$ the structure constants of the algebra $\mathcal{A}$ with respect to the basis $\left\{x_{V}\right\}$, i.e., $x_{U} x_{V}=$ $\sum_{W} N_{U V}^{W} x_{W}$, then it suffices to show $\langle U\rangle\langle V\rangle=\sum_{W} N_{U V}^{W}\langle W\rangle$. Since

$$
\begin{aligned}
N_{U V}^{W} & =\left\langle x_{U} x_{V} \mid x_{W}\right\rangle=\frac{1}{|\Gamma|} \sum_{x, y, z \in X} \sum_{\gamma \in \Gamma(x, y, z)} \chi_{U}^{(x, y)}(\gamma) \chi_{V}^{(y, z)}(\gamma) \overline{\chi_{W}^{(x, z)}(\gamma)} \\
\chi_{U}^{(x, y)}(\gamma) & =\chi_{U}^{\left(x \gamma_{0}, y \gamma_{0}\right)}\left(\gamma_{0}^{-1} \gamma \gamma_{0}\right),
\end{aligned}
$$

we have

$$
N_{U V}^{W}=\frac{1}{|\Gamma|} \sum_{l=1}^{d}\left|\Gamma / \Gamma\left(x_{l}, y_{l}, z_{l}\right)\right| \sum_{\gamma \in \Gamma\left(x_{l}, y_{l}, z_{l}\right)} \chi_{U}^{\left(x_{l}, y_{l}\right)}(\gamma) \chi_{V}^{\left(y_{l}, z_{l}\right)}(\gamma) \overline{\chi_{W}^{\left(x_{l}, z_{l}\right)}(\gamma)}
$$

where $\left\{\left(x_{l}, y_{l}, z_{l}\right) ; l=1, \cdots, d\right\}$ is a set of representatives for $\Gamma$-orbits on the set

$$
\left\{(H a, H b, H c) \in X \times X \times X ; a b^{-1} \in \mathbf{s}(U), b c^{-1} \in \mathbf{s}(V), a c^{-1} \in \mathbf{s}(W)\right\} .
$$

Therefore, by Theorem $3, N_{U V}^{W}$ coincides with the multiplicity of the trivial bundle in $U \otimes_{H} V \otimes_{H} W^{*}$, which is the same as the multiplicity of $W$ in $U \otimes_{H} V$.

Corollary 5. The fusion algebra ${ }_{H} \mathcal{F}_{H}$ is ${ }^{*}$-isomorphic to a multi-matrix algebra. Moreover, ${ }_{H} \mathcal{F}_{H}$ is commutative if and only if the action of the centralizer $C_{\Gamma}\left(\gamma_{\alpha}\right)$ on $X\left(\gamma_{\alpha}\right)$ is multiplicity-free.

Let $\alpha$ be an outer action of a finite group $H$ on the hyperfinite $\mathrm{II}_{1}$-factor $R_{0}$. Then, the asymptotic inclusion of $R_{0} \subseteq R_{0} \rtimes_{\alpha} H$ is of the form: $P^{H \times H} \subseteq$ $P^{H}$, where $H$ means the diagonal subgroup of $H \times H$ (see $[\mathbf{1 6}, \mathbf{3 0}]$ ). It was announced by Ocneanu and shown in [7] that the fusion algebra of an asymptotic inclusion is always commutative.

Example. Let $\Gamma=H \times H$ with $H$ diagonally imbedded into $\Gamma$. In this case, a generalization of the fusion algebra $\mathcal{A}$ using a 3-cocycle of $H$ is known 
as the fusion algebra of an orbifold model $([\mathbf{1}, \mathbf{2}, \mathbf{5}, \mathbf{3 5}])$. In [26], Lusztig constructed the algebra $\mathcal{A}$ from $\operatorname{Ad} H$-bundles on $H$. It is worth noting that the algebra $\mathcal{A}$ in this case is commutative.

Let $C_{0}, \ldots, C_{d}$ be the conjugacy classes of $H$ so that those of $\Gamma=H \times H$ are $C_{i} \times C_{j}(i, j=0,1, \ldots, d)$. Notice that $X(\gamma) \neq \emptyset$ if and only if $\gamma$ belongs to $C_{i} \times C_{i}$ for some $i$. Thus,

$$
\mathcal{A} \cong \bigoplus_{i=0}^{d}\left\{x \in \mathcal{B}\left(\ell^{2}\left(X\left(h_{i}, h_{i}\right)\right)\right) ; x \gamma=x \text { for all } \gamma \in C_{H}\left(h_{i}\right) \times C_{H}\left(h_{i}\right)\right\} .
$$

Here, $h_{i} \in C_{i}$ and $C_{H}(h)$ denotes the centralizer of $h$ in $H$. Identifying $H \backslash \Gamma$ with $H$ by the correspondence $H(a, b) \in H \backslash \Gamma \longleftrightarrow a^{-1} b \in H$, we can see

$$
\mathcal{A} \cong \bigoplus_{i=0}^{d} \mathcal{Z}\left(\mathbb{C}\left[C_{H}\left(h_{i}\right)\right]\right)
$$

where $\mathcal{Z}\left(\mathbb{C}\left[C_{H}\left(h_{i}\right)\right]\right)$ denotes the center of the group algebra $\mathbb{C}\left[C_{H}\left(h_{i}\right)\right]$. In particular, $\mathcal{A}$ is commutative.

The quantum double of the group Hopf algebra $\ell^{\infty}(H)$ of the finite group $H$ can be formally defined as the vector space $\mathcal{D}$ with a basis $(h, k)(h, k \in H)$ and the multiplication rule

$$
(h, k)\left(h^{\prime}, k^{\prime}\right)=\delta_{k, h k^{\prime} h^{-1}}\left(h h^{\prime}, k\right)
$$

(see [6]). One can easily check that the center of $\mathcal{D}$ is the fixed-point algebra (under the diagonal adjoint action by $H$ ) of the subalgebra spanned by $\left\{\left(h, h^{\prime}\right) ; h h^{\prime}=h^{\prime} h\right\}$ (see [27]). It is plain to see that the latter is isomorphic to the direct sum (1). Therefore, we have shown that in the present case the fusion algebra of the asymptotic inclusion is actually isomorphic to the center of the quantum double of the group Hopf algebra $\ell^{\infty}(H)$.

\section{Hecke Algebras.}

Hecke operators are considered in $[40,41]$ as operators acting on character rings of subgroups of some ambient group $\Gamma$. In this part, we shall recognize them as elements in the fusion algebra $\mathcal{F}$ of vector bundles over $\Gamma$.

Let us begin with some of relevant definitions: Let $H, K$ be finite subgroups of $\Gamma$ and consider $X=H \backslash \Gamma$ as a right $K$-space. For $x \in X$, let $K(x)=\{k \in K ; x k=x\}$ be the isotropy group of the $K$-action. We consider the set

$$
\Xi=\{(x, \xi) ; x \in X \text { and } \xi \text { is an irreducible character of } K(x)\}
$$


in which an equivalence relation $\sim$ is defined by $(x, \xi) \sim(y, \eta)$ if and only if $x k=y$ and $\xi^{k}=\xi\left(k \cdot k^{-1}\right)=\eta$ for some $k \in K$. For $(x, \xi) \in \Xi$, the equivalence class containing $(x, \xi)$ is denoted by $[x, \xi] \in$ $\Xi / \sim$. Let ${ }_{H} \mathcal{H}_{K}$ be a complex vector space generated by $\Xi / \sim$, and for $[x, \xi] \in{ }_{G} \mathcal{H}_{H}$ and $[y, \eta] \in{ }_{H} \mathcal{H}_{K}$ we define the product $[x, \xi][y, \eta] \in{ }_{G} \mathcal{H}_{K}$ by

$$
[G a, \xi][H b, \eta]=\sum_{\dot{h} \in G^{a} \cap H \backslash H / H \cap^{b} K}\left[G a h b,\left(\xi^{h b} \eta\right)_{G^{a h b} \cap H^{b} \cap K}{ }^{G^{a h b} \cap K}\right] .
$$

Here, ${ }^{b} K=b K b^{-1}, G^{a}=a^{-1} G a$ and so on. The character on the right hand side is obtained by first taking restriction to $G^{a h b} \cap H^{b} \cap K$ and then inducing up to $G^{a h b} \cap K$.

With this operation, the totality of vector spaces $\left\{{ }_{H} \mathcal{H}_{K}\right\}$ is made into an associative algebra.

Now take an $H-K$ bundle $V$ over $\Gamma$ and define the element $\theta_{V}$ in ${ }_{H} \mathcal{H}_{K}$ by

$$
\theta_{V}=\sum_{H \gamma K \in H \backslash \mathbf{s}(V) / K}[H \gamma, \xi]
$$

Here, $\xi$ denotes the character of $K(H \gamma)=\left(\gamma^{-1} H \gamma\right) \cap K$ obtained by taking trace of

$$
v \in V_{\gamma} \mapsto\left(\gamma k \gamma^{-1}\right) v k^{-1} \in V_{\gamma} .
$$

Note that the stabilizer $H \times_{\gamma} K=\left\{(h, k) \in H \times K ; h \gamma k^{-1}=\gamma\right\}$ of the $H-K$ action is isomorphic to $K(H \gamma)$ by $(h, k) \mapsto k$.

Theorem 6. For each $H, K$,

$$
\langle V\rangle \in{ }_{H} \mathcal{F}_{K} \mapsto \theta_{V} \in{ }_{H} \mathcal{H}_{K}
$$

is well-defined and gives rise to an isomorphism of vector spaces. Furthermore, this isomorphism preserves multiplication in the sense that

$$
\theta_{U \otimes_{H} V}=\theta_{U} \theta_{V}
$$

where $U$ is a $G-H$ bundle over $\Gamma$.

Proof. First note that $\theta_{V}$ depends on $V$ only through the equivalence class

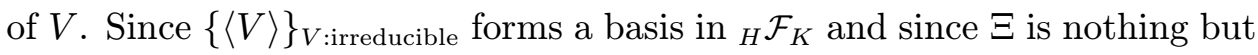
$\left\{\theta_{V} ; V\right.$ is irreducible $\}$, the correspondence in question is well-defined and gives rise to an isomorphism.

It remains to show the multiplicativity. Let $U$ and $V$ be as in the statement of the theorem. For the proof of this property, we may assume that $U$ 
and $V$ are irreducible. Let $\mathbf{s}(U)=G a H$ and $\mathbf{s}(V)=H b K$ be their supports. Recall that the $G-K$ bundle $U \otimes_{H} V$ is defined as the pushforward of the vector bundle $U \otimes_{H} V$ over the set $\mathbf{s}(U) \times_{H} \mathbf{s}(V)$ which is the quotient bundle of the outer tensor product bundle $U \otimes V$. Thus $U \otimes_{H} V$ is first decomposed through $G-K$ orbits in $U \bigotimes_{H} V$

$$
G \curvearrowright \underset{\mathbf{s}(U) \times_{H} \mathbf{s}(V)}{\substack{\otimes_{H} V \\ \downarrow}} \curvearrowleft K .
$$

It is easy to check that the map $h \in H \mapsto G\left(a h \times_{H} b\right) K \in G \backslash \mathbf{s}(U) \times_{H} \mathbf{s}(V) / K$ induces a bijection

$$
a^{-1} G a \cap H \backslash H / H \cap b K b^{-1} \cong G \backslash \mathbf{s}(U) \times_{H} \mathbf{s}(V) / K .
$$

Choose a double coset in $a^{-1} G a \cap H \backslash H / H \cap b K b^{-1}$ with a representative $h \in H$ and set $c=a h \times_{H} b \in \mathbf{s}(U) \times_{H} \mathbf{s}(V)$. Note that $\left(U \otimes_{H} V\right)_{c}=$ $U_{a h} \otimes_{H} V_{b}=U_{a} \otimes_{H} V_{h b}$. Let $W$ be a $G-K$ bundle supported by $G a h b K$ which is obtained as the pushforward of the $G-K$ orbit in $U \otimes_{H} V$ containing $\left(U \otimes_{H} V\right)_{c}$. Then the fibre of $W$ at $a h b \in \Gamma$ is given by the direct sum of $\left\{g \times_{H} k \in G \times_{H} K ; g k=a h b\right\} \cap G c K$, i.e.,

$$
W_{a h b}=\bigoplus_{(g, k) \in\left(G \times_{a h b} K\right) /\left(G \times_{c} K\right)} g\left(U \otimes_{H} V\right)_{c} k^{-1} .
$$

Here, $G \times \times_{a h b} K$ and $G \times{ }_{c} K$ are stabilizers of $G$ - $K$ action at $a h b \in \Gamma$ and $c \in G \times{ }_{H} K$ respectively. Recall that $G \times{ }_{c} K=\left\{(g, k) \in G \times K ; g a h \times{ }_{H} b k^{-1}=\right.$ $\left.a h \times_{H} b\right\}$ and similarly for $G \times{ }_{a h b} K$. Explicitly these groups are given by

$$
G \times{ }_{a h b} K=\left\{\left(a h b k(a h b)^{-1}, k\right) ; k \in(a h b)^{-1} G a h b \cap K\right\}
$$

and

$$
G \times{ }_{c} K=\left\{\left(a h b k(a h b)^{-1}, k\right) ; k \in(a h b)^{-1} G a h b \cap b^{-1} H b \cap K\right\} .
$$

In this way, the representation of the stabilizer $G \times \times_{a h b} K$ at $W_{a h b}$ is $\operatorname{Ind}_{G \times c}^{G \times \times_{a h b} K}\left(U_{a h} \otimes_{H} V_{b}\right)$.

Let $\xi$ be the character of $U$ at a (i.e., a character of $H(G a)$ ), $\xi^{\prime}$ the character of $U$ at $a h$, and $\eta$ the character of $V$ at $b$. Note that $\xi^{\prime}=\xi^{h}=$ $\xi\left(h \cdot h^{-1}\right)$.

Then the character of $W$ at $a h b$ is induced from the character $\zeta$ of $G \times{ }_{a h b} K$ which is calculated as follows:

$$
u^{\prime} \otimes_{H} v \in U_{a h} \otimes_{H} V_{b} \mapsto a h b k(a h b)^{-1} u^{\prime} \otimes_{H} v k^{-1}
$$




$$
\begin{aligned}
& =a h b k(a h b)^{-1} u^{\prime}\left(b k^{-1} b^{-1}\right) \otimes_{H}\left(b k^{-1} b^{-1}\right)^{-1} v k^{-1} \\
& =(a h)\left(b k b^{-1}\right)(a h)^{-1} u^{\prime}\left(b k b^{-1}\right)^{-1} \otimes_{H} b k b^{-1} v k^{-1},
\end{aligned}
$$

i.e., taking trace, we have

$$
\zeta(k)=\xi^{\prime}\left(b k b^{-1}\right) \eta(k)=\xi\left(h b k b^{-1} h^{-1}\right) \eta(k)=\xi^{h b}(k) \eta(k)
$$

for $k \in(a h b)^{-1} G a h b \cap b^{-1} H b \cap K$.

\section{Automorphisms appearing in bimodules.}

Throughout the section, let $M \supseteq N$ be an irreducible factor-subfactor pair of finite index and finite depth with the Jones tower $([\mathbf{8}, \mathbf{1 4}])$

$$
N \subseteq M=M_{0} \subseteq M_{1} \subseteq M_{2} \subseteq M_{3} \subseteq \cdots .
$$

Let $\mathcal{H}\left(={ }_{N} \mathcal{H}_{M}\right)$ be the basic $N-M$ bimodule ${ }_{N} L^{2}(M)_{M}$ as in 2.2. Each automorphism $\alpha \in \operatorname{Aut}(M)$ gives rise to the $M-M$ bimodule ${ }_{\alpha} \mathcal{H}$ (of dimension 1):

$$
{ }_{\alpha} \mathcal{H}=L^{2}(M) \quad \text { with } \quad m_{1} \cdot \xi \cdot m_{2}=\alpha\left(m_{1}\right) J_{M} m_{2}^{*} J_{M} \xi .
$$

Let $u$ be the canonical implementation of $\alpha$. Notice

$$
u^{*}\left(\alpha\left(m_{1}\right) J_{M} m_{2}^{*} J_{M} \xi\right)=m_{1} J_{M} \alpha^{-1}\left(m_{2}^{*}\right) J_{M} u^{*} \xi
$$

because $u$ commutes with the modular conjugation $J_{M}$. This means that the $M-M$ bimodule ${ }_{\alpha} \mathcal{H}$ is unitarily equivalent to (hence identified with) $\mathcal{H}_{\alpha^{-1}}$ defined by

$$
\mathcal{H}_{\alpha^{-1}}=L^{2}(M) \quad \text { with } \quad m_{1} \cdot \xi \cdot m_{2}=m_{1} J_{M} \alpha^{-1}\left(m_{2}^{*}\right) J_{M} \xi .
$$

Conversely, any $M-M$ bimodule of dimension 1 arises in this way. We also remark that an inner perturbation of $\alpha$ does not change the unitary equivalence class of the above bimodule. In what follows unitarily equivalent bimodules will be identified.

As in $[29,30,36,37]$ we consider the $M$ - $M$ bimodules

${ }_{M} L^{2}(M)_{M}, \mathcal{H}^{*} \otimes_{N} \mathcal{H}={ }_{M} L^{2}\left(M_{1}\right)_{M}, \mathcal{H}^{*} \otimes_{N} \mathcal{H} \otimes_{M} \mathcal{H}^{*} \otimes_{N} \mathcal{H}={ }_{M} L^{2}\left(M_{2}\right)_{M}, \cdots$

(which correspond to the "half" of the second sequence in 2.4). The $M-M$ bimodules of dimension 1 appearing in the irreducible decomposition obviously form a group (with respect to the relative tensor product $\cdots$ see also Remark before Lemma 9). The corresponding automorphisms are determined up to inner perturbations as remarked above. They may or may not be chosen from $\operatorname{Aut}(M, N)=\{\theta \in \operatorname{Aut}(M) ; \theta(N)=N\}$. 
Proposition 7 ([19, Proposition 4]). One can find a unitary $u$ in $M$ satisfying $\operatorname{Ad} u \circ \alpha \in \operatorname{Aut}(M, N)$ if and only if there exists an $N-N$ bimodule $\mathcal{K}$ of dimension 1 such that the induced $M-N$ bimodule $\mathcal{H}^{*} \otimes_{N} \mathcal{K}$ of $\mathcal{K}$ is contragredient to the restricted $N-M$ bimodule $\mathcal{H} \otimes_{M \alpha} \mathcal{H}$ of ${ }_{\alpha} \mathcal{H}$. Furthermore, if ${ }_{\alpha} \mathcal{H} \prec{ }_{M} L^{2}\left(M_{k}\right)_{M}$, then $\mathcal{K} \prec{ }_{N} L^{2}\left(M_{k}\right)_{N}$.

For the Haagerup subfactor with index $(5+\sqrt{13}) / 2([9])$, one obtains two (non-trivial) $M-M$ bimodules with dimension 1 (i.e., automorphisms) in ${ }_{M} L^{2}\left(M_{3}\right)_{M}$. However, these cannot be adjusted to automorphisms in $\operatorname{Aut}(M, N)$. Actually one obtains an abundance of such examples from (finite) group-subgroup pairs (see Theorem 10). For example, we have this phenomenon for the pair $M^{A_{4}} \supseteq M^{A_{5}}$ (of the fixed-point algebras by an alternating group action), and this happens because the two non-trivial characters of $A_{4} \cong\left(\mathbf{Z}_{2} \times \mathbf{Z}_{2}\right) \rtimes \mathbf{Z}_{3}$ (i.e., those arising from $\mathbf{Z}_{3}$ ) cannot be lifted to characters of the simple group $A_{5}$ (see Figures 3,4 in [21]).

We now look at automorphisms $\alpha \in \operatorname{Aut}(M, N)$ such that ${ }_{\alpha} \mathcal{H} \prec \bigsqcup_{k}{ }_{M} L^{2}\left(M_{k}\right)_{M}$. This containment is equivalent to the existence of a non-zero $x \in M_{k}$ (for some $k$ ) satisfying

$$
n x=x \alpha(n) \text { for } n \in N .
$$

An automorphism with this property is called non-strongly outer $([\mathbf{3}, \mathbf{1 9}])$ and plays an important role in the study of automorphisms for $M \supseteq N$. Analytic aspect of such an automorphism (i.e., the central triviality) was investigated and the related important invariant $\chi(M, N)$ was studied by Kawahigashi ([15], see also [32]).

When $\left.\alpha\right|_{N}=\operatorname{Id}_{N}$, it is obviously non-strongly outer, and $\operatorname{Gal}(M, N)=$ $\{\alpha \in \operatorname{Aut}(M) ; \alpha(n)=n$ for $n \in N\}$ is known as the Galois group of $M \supseteq$ $N$ (see for example [17]). The next result may be a folk result among specialists.

Proposition 8. The $M-M$ bimodules of dimension 1 in ${ }_{M} L^{2}\left(M_{1}\right)_{M}$ form a finite group, and this group is exactly $\operatorname{Gal}(M, N)$.

The irreducibility assumption of $M \supseteq N$ is essential here. To see this, let

$$
M=P \otimes M_{2}(\mathbf{C}) \supseteq N=\left\{\left(\begin{array}{cc}
x & 0 \\
0 & \alpha(x)
\end{array}\right) ; x \in P\right\}
$$

be the locally trivial inclusion arising from an outer automorphism $\alpha \in$ Aut $(P)$. We assume that the outer period of $\alpha$ is not 2. Then, we easily see that $\mathcal{H}^{*} \otimes_{N} \mathcal{H}$ decomposes into four one dimensional bimodules. The identity bimodule ${ }_{M} L^{2}(M)_{M}$ appears twice, and the other two are $\alpha_{\alpha \otimes \operatorname{Id}_{M_{2}(\mathbf{C})}} \mathcal{H}$ and $\alpha^{-1} \otimes \operatorname{Id}_{M_{2}(\mathbf{C})} \mathcal{H}$. These three automorphisms do not form a group, and the two 
non-trivial automorphisms do not belong to $\operatorname{Gal}(M, N)$ either. (Actually, routine computations show that $\operatorname{Gal}(M, N)$ consists of just inner automorphisms in this case.)

In the properly infinite case the above result means that $\operatorname{Gal}(M, N)$ consisits of automorphisms appearing in Longo's canonical endomorphism $\gamma([\mathbf{2 3}])$ attached to $M \supseteq N$ (see $[\mathbf{1 2}, \mathbf{2 4}])$.

Proof. The group property follows from Lemma 9 below (see also Remark).

The bimodule $\mathcal{H} \otimes_{M}{ }_{\alpha} \mathcal{H}=\mathcal{H} \otimes_{M} \mathcal{H}_{\alpha^{-1}}$ is obviously $L^{2}(M)$ equipped with the following $N-M$ action:

$$
n \cdot \xi \cdot m=n J_{M} \alpha^{-1}\left(m^{*}\right) J_{M} \xi .
$$

By the same calculation as (3), it is equivalent to $\mathcal{K}=L^{2}(M)$ equipped with the $N-M$ action

$$
n \cdot \xi \cdot m=\alpha(n) J_{M} m^{*} J_{M} \xi .
$$

By Lemma $9,{ }_{\alpha} \mathcal{H} \prec \mathcal{H}^{*} \otimes_{N} \mathcal{H}$ if and only if $\mathcal{H}=\mathcal{H} \otimes_{M}{ }_{\alpha} \mathcal{H}=\mathcal{K}$. The latter condition means that there is a unitary $u$ (on $L^{2}(M)$ ) intertwining the right $M$-action (i.e., $u \in M$ ) and intertwining the left $N$-action, that is,

$$
u n=\alpha(n) u \quad(\text { for all } n \in N) .
$$

Hence, ${ }_{\alpha} \mathcal{H} \prec \mathcal{H}^{*} \otimes_{N} \mathcal{H}$ if and anly if one finds a unitary $u \in M$ satisfying $\left.\operatorname{Ad} u \circ \alpha\right|_{N}=\operatorname{Id}_{N}$.

Remark. As in the above proof, for automorphisms $\alpha, \beta \in \operatorname{Aut}(M)$ the relative tensor product ${ }_{\alpha} \mathcal{H} \otimes_{M} \mathcal{H}_{\beta}$ can be identified with $L^{2}(M)$ with equipped with the $M-M$ action

$$
m_{1} \cdot \xi \cdot m_{2}=\alpha\left(m_{1}\right) J_{M} \beta\left(m_{2}^{*}\right) J_{M} \xi
$$

The above right hand side is equal to

$$
v\left(v^{*} \alpha\left(m_{1}\right) v J_{M} m_{2}^{*} J_{M}\left(v^{*} \xi\right)\right)=v\left(\beta^{-1} \circ \alpha\left(m_{1}\right) J_{M} m_{2}^{*} J_{M}\left(v^{*} \xi\right)\right),
$$

where $v$ denotes the canonical implementation of $\beta$. Therefore, we have shown ${ }_{\alpha} \mathcal{H} \otimes_{M} \mathcal{H}_{\beta}={ }_{\beta^{-1} \circ \alpha} \mathcal{H}$, that is, we have

$$
\begin{aligned}
{ }_{\alpha} \mathcal{H} \otimes_{M}{ }_{\beta} \mathcal{H} & ={ }_{\beta \circ \alpha} \mathcal{H} \\
\mathcal{H}_{\alpha} \otimes_{M} \mathcal{H}_{\beta} & =\mathcal{H}_{\alpha \circ \beta}
\end{aligned}
$$

(see Proposition 2.1, [13]). 
Lemma 9 ([36, Theorem 1.5]). $\mathcal{H} \otimes_{M \alpha} \mathcal{H}=\mathcal{H}$ if and only if $\mathcal{H} \prec \mathcal{H}^{*} \otimes_{N} \mathcal{H}$.

Proof. We have ${ }_{\alpha} \mathcal{H} \prec \mathcal{H}^{*} 1 \otimes_{N} \mathcal{H}$ if and only only if $\mathcal{H} \prec \mathcal{H} \otimes_{M}{ }_{\alpha} \mathcal{H}$ by the Frobenius reciprocity. The result thus follows from the obvious irreducibility of $\mathcal{H} \otimes_{M \alpha} \mathcal{H}$.

So far we have introduced the three groups: (i) The group of all $M-M$ bimodules of dimension 1 appearing in $\bigsqcup_{k}{ }_{M} L^{2}\left(M_{k}\right)_{M}$, (ii) the group of $M-M$ bimodules of dimension 1 appearing in $\bigsqcup_{k}{ }_{M} L^{2}\left(M_{k}\right)_{M}$ and satisfying the condition in Proposition 7 (i.e., the group of non-strongly outer automorphisms modulo the inner automorphisms), (iii) the Galois group $\operatorname{Gal}(M, N)$. As mentioned above, $\operatorname{Gal}(M, N)$ is the smallest among the three (see also Proposition 8). Let $G \supseteq H$ be a group-subgroup pair, and we choose and fix an outer action on a factor $P$ (as in 2.4). Therefore, we have the pairs $P \rtimes G \supseteq P \rtimes H, P^{H} \supseteq P^{G}$ of factors. In the rest of the section, we will explicitly compute the three groups for these pairs.

We begin with $M=P \rtimes G \supseteq N=P \rtimes H$. The irreducible $M-M$ bimodules and $M-N$ bimodules (similarly the $N-M$ bimodules) are parameterized by $\hat{G}$ and $\hat{H}$ respectively while the irreducible $N-N$ bimodules are parameterized by $\bigsqcup_{i} \hat{H}_{i}$ as was explained in 2.4. Here, $\left\{g_{i}\right\}_{i=1,2, \cdots, n}$ is a complete set of representatives for $H \backslash G / H$ (with $g_{1}=1$ ) and $H_{i}=g_{i} H g_{i}^{-1} \cap H$. It is easy to see that the relative tensor products among $M-M$ bimodules are described by the decomposition rule for the tensor products of corresponding irreducible representations, and details are left to the reader. On the other hand, the (much more subtle) relative tensor products among $N-N$ bimodules were studied in the previous sections.

The index formula in [22] (with $\Gamma=G$ and $H \subseteq G$ ) shows that the indices (i.e., the squares of dimensions) of $M-M, M-N$ (or $N-M$ ), and $N-N$ bimodules are

$$
\begin{array}{cc}
\frac{(\operatorname{deg} \pi \times|G|)^{2}}{|G| \times|G|}=(\operatorname{deg} \pi)^{2} & \text { for } \pi \in \hat{G} \\
\frac{(\operatorname{deg} \pi \times|G|)^{2}}{|G| \times|H|}=(\operatorname{deg} \pi)^{2} \times|G / H| & \text { for } \pi \in \hat{H} \\
\frac{\left(\operatorname{deg} \pi \times\left|H g_{i} H\right|\right)^{2}}{|H| \times|H|}=(\operatorname{deg} \pi)^{2} \times\left|H / H_{i}\right| & \text { for } \quad \pi \in \hat{H}_{i}
\end{array}
$$

respectively. Here, deg $\pi$ means the dimension of the irreducible representation $\pi$, and the above numerators are the squares of the dimensions of the relevant $(G-G, G-H$, and $H-H)$ bundles. The last equality holds because of $\left|H g_{i} H\right|=|H \times H| /\left|H_{i}\right|$.

From the first formula the one dimensional $M-M$ bimodules are exactly those coming from the (one dimensional) characters $\pi$ of $G$. Also the third 
formula says that the $N-N$ bimodule arising from $\pi^{\prime} \in \hat{H}_{i}$ is one dimensional if and only if

$$
\operatorname{deg} \pi^{\prime}=1 \text { and } H_{i}=H .
$$

In the first case the corresponding automorphisms $\alpha_{\pi}$ (of $M$ ) can be always chosen to leave $N$ globally invariant. This can be checked by Proposition 7 . Namely, the restriction of $\pi$ to $H$ should be contragredient to $\operatorname{Ind}_{H_{i}=H}^{H} \pi^{\prime}$ for some $i$ with $H_{i}=H$ and $\pi^{\prime} \in \hat{H}_{i}$ with deg $\pi^{\prime}=1$ (recall the last paragaph in 2.4). However, this is obviously satisfied since $g_{1}=1$ and $H_{1}=H$. It is also possible to see this fact from the explicit construction of $\alpha_{\pi}$ in [22]:

$$
\alpha_{\pi}: \sum_{g \in G} x_{g} \lambda_{g} \in M=P \rtimes G \longrightarrow \sum_{g \in G} \pi(g) x_{g} \lambda_{g} \in M .
$$

Therefore, the groups (i), (ii) are both the group of characters of $G$ (i.e., $G /[G, G])$. The above automorphism $\alpha_{\pi}$ fixes $H$ element-wise if and only if $\left.\pi\right|_{H}=\operatorname{Id}_{H}$. Hence, $\operatorname{Gal}(M, N)$ consists of the characters vanishing on $H$ (as expected $\cdots$ see [17] for example).

The other case $M=P^{H} \supseteq N=P^{G}$ is actually more interesting. Notice that the roles of $M-M$ and $N-N$ bimodules are switched now. Hence, in the rest of the section we will study one dimensional $N-N$ bimodules (in the crossed product case). The second condition of (4) means that $g_{i}$ normalizes the subgroup $H$ :

$$
\left.g_{i} H g_{i}^{-1}=H \quad \text { (i.e., } \quad g_{i} \in \mathcal{N}_{G}(H)\right) .
$$

Notice that, for $g_{i}, g_{j} \in \mathcal{N}_{G}(H)$, we have $H g_{i} H=H g_{j} H$ if and only if $g_{i} \in g_{j} H$. Therefore, $H_{i}$ 's satisfying $H=H_{i}$ are parameterized by the quotient group $\mathcal{N}_{G}(H) / H$ (which will be referred to as the Weyl group). Thus, we have seen that the one dimensional $N-N$ bimodules are parameterized by the product space $\Pi \times\left(\mathcal{N}_{G}(H) / H\right)$. Here, $\Pi(\cong H /[H, H])$ denotes the group of characters of $H$. The condition in (the dual version of) Proposition 7 is satisfied when $\operatorname{Ind}_{H_{i}=H}^{H} \pi=\pi$ is the restriction (to $H$ ) of some one dimensional representation of $G$ (the last paragraph in 2.4), that is, when $\pi$ is extended to a character of $G$. Denoting all extendable characters (on $H$ ) by $\Pi_{0}$, we conclude that the group in (ii) is parameterized by the product space $\Pi_{0} \times\left(\mathcal{N}_{G}(H) / H\right)$.

So far we have not touched the group structure. Let $g, g_{0}$ be in $\mathcal{N}_{G}(H)$, and $U, U_{0}$ be $H-H$ bundles (supported on $\mathbf{s}(U)=H g H, \mathbf{s}\left(U_{0}\right)=H g_{0} H$ ) corresponding to characters $\pi, \pi_{0} \in \hat{H}$ respectively. Theorem 6 shows that the product bundle $U \otimes_{H} U_{0}$ is supported on $H g g_{0} H$ (and hence indexed by $\left.g g_{0} \in \mathcal{N}_{G}(H)\right)$ with the corresponding (one-dimensional) representation:

$$
h \in H \longrightarrow \pi^{g_{0}}(h) \pi_{0}(h) \in \mathbf{T} .
$$


In fact, in (2) (where all of $G, H, K$ are $H$ and $\Gamma=G$ ) the right hand side reduces to a single term and the restriction-induction procedure disappears because of $g, g_{0} \in \mathcal{N}_{G}(H)$. Notice that $g \in \mathcal{N}_{G}(H)$ naturally acts on $\Pi$, the group of characters on $H$, via

$$
h \in H \longrightarrow \pi^{g}(h)=\pi\left(g h g^{-1}\right) \in \mathbf{T} .
$$

Since

$$
\pi\left(k h k^{-1}\right)=\pi(k) \pi(h) \overline{\pi(k)}=\pi(h)
$$

for $k \in H$, we get the action of $\mathcal{N}_{G}(H) / H$ on $\Pi$. The formula (5) of course corresponds to the multiplication rule in the semi-direct product $\Pi \rtimes\left(\mathcal{N}_{G}(H) / H\right)$. Also, notice that $\mathcal{N}_{G}(H) / H$ acts trivially on $\Pi_{0}$, the extendable characters, by the same computation as (6).

Summing up the arguments so far, we have proved:

Theorem 10. Assume $M=P^{H} \supseteq N=P^{G}$, the fixed-point algebra factors.

(i) The group of one dimensional $M-M$ bimodules in $\bigsqcup_{k}{ }_{M} L^{2}\left(M_{k}\right)_{M}$ is the semi-direct product $\Pi \rtimes\left(\mathcal{N}_{G}(H) / H\right)$.

(ii) The group of non-strongly outer automorphisms for $M \supseteq N$ modulo the inner automorphisms is the product group $\Pi_{0} \times\left(\mathcal{N}_{G}(H) / H\right)$.

Notice that the Weyl group $\mathcal{N}_{G}(H) / H$ is included in both of the two groups. We now recall the algorithm in the last paragraph in 2.4. The irreducible $N-N$ bimodules in $\mathcal{H}^{*} \otimes_{N} \mathcal{H}$ are given by the trivial representations $\left\{\operatorname{Id}_{H_{i}}\right\}_{i=1,2, \cdots, n}$. The $N-N$ bimodule corresponding to $\operatorname{Id}_{H_{i}} \in \hat{H}_{i}$ is one dimensional if and only if $H_{i}=H((4))$. Consequently, the Weyl group $\mathcal{N}_{G}(H) / H$ is exactly $\operatorname{Gal}(M, N)$ by Proposition 8 .

\section{References}

[1] P. Bantay, Orbifolds and Hopf algebra, Phys. Lett., 245(B) (1990), 477-479.

[2] - Orbifolds, Hopf algebras and the Moonshine, Lett. Math. Phys., 22 (1991), 187-194.

[3] M. Choda and H. Kosaki, Strongly outer actions for an inclusion of factors, J. Funct. Anal., 122 (1994), 315-332.

[4] R. Dijkgraaf, V. Pasquier and R. Roche, Quasi Hopf algebras, group cohomology and orbifold models, Nucl. Phys. B (Proc. Suppl.), 18B (1990), 60-72.

[5] R. Dijkgraaf, C. Vafa, E. Verlinde and H. Verlinde, The operator algebras of orbifold models, Commun. Math. Phys., 123 (1989), 485-526.

[6] V. Drinfeld, Quantum groups, Proc. ICM at Berkeley, Amer. Math. Soc., 1986. 
[7] D. Evans and Y. Kawahigashi, On Ocneanu's theory of asymptotic inclusions for subfactors, topological quantum field theories, and quantum doubles, Internat. J. Math., 6 (1995), 205-228.

[8] F. Goodman, de la Harpe and V.F.R. Jones, Coxeter Dynkin Diagrams and Towers of Algebras, Springer, 1989.

[9] U. Haagerup, Principal graphs of subfactors in the index range $4<[M: N]<$ $3+\sqrt{2}$, in "Subfactors", World Scientific, (1994), 1-38.

[10] J.F. Havet, Espérance conditionnelle minimale, J. Operator Theory, 24 (1990), $33-55$.

[11] F. Hiai, Minimizing indices of conditional expectations onto a subfactor, Publ. RIMS, Kyoto Univ., 24 (1988), 673-678.

[12] M. Izumi, Applications of fusion rules to classification of subfactors, Publ. RIMS, Kyoto Univ., 27 (1991), 953-994.

[13] , Goldman's type theorem for index 3, Publ. RIMS, Kyoto Univ., 28 (1992), 833-843.

[14] V.F.R. Jones, Index of subfactors, Invent. Math., 66 (1983), 1-25.

[15] Y. Kawahigashi, Centrally trivial automorphisms and an analogue of Connes' $\chi(M)$ for subfactors, Duke Math. J., 71 (1993), 93-118.

[16] Y. Kawahigashi, Orbifold subfactors, central sequences, and the relative Jones invariant $\kappa$, IMRN, 3 (1995), 129-140.

[17] S. Kawakami and H. Yoshida, The constituents of Jones' index analyzed from the structure of the Galois theory, Math. Japonica, 33 (1988), 551-557.

[18] H. Kosaki, Extension of Jones' theory on index to arbitrary factors, J. Funct. Anal., 66 (1986), 123-140.

[19] - Automorphisms in the irreducible decomposition of sectors, in "Quantum and Non-commutative Analysis", Kluwer Academic, (1993), 305-316.

[20] H. Kosaki and R. Longo, A remark on the minimal index of subfactors, J. Funct. Anal., 107 (1992), 458-470.

[21] H. Kosaki and T. Sano, Non-splitting inclusions of factors of type $\mathrm{III}_{0}$, to appear in Pacific J. Math.

[22] H. Kosaki and S. Yamagami, Irreducible bimodules associated with crossed product algebras, Internat. J. Math., 3 (1992), 661-676.

[23] R. Longo, Simple injective factors, Adv. in Math., 63 (1987), 152-172.

[24] Index of subfactors and statistics of quantum fields II, Commun. Math. Phys., 130 (1990), 285-309.

[25] _ Minimal index and braided subfactors, J. Funct. Anal., 109 (1992), 98-112.

[26] G. Lusztig, Leading coefficients of character values of Hecke algebras, in "The Arcata Conference on Representation of Finite Groups", Proc. Sympo. Pure Math., Vol. 47 (1987), Amer. Math. Soc., 235-262.

[27] G. Mason, The quantum double of a finite group and its rôle in conformal field theory, in "Groups '93 Galway/St. Andrews", Vol. 2, Cambridge Univ. Press, Cambridge, (1995), 405-417.

[28] A. Munemasa, On some fusion algebra attached to finite group action, unpublished note. 
[29] A. Ocneanu, Quantized groups, string algebras, and Galois theory, in "Operator Algebras and Applications Vol. II", Cambridge Univ. Press, 1988.

[30] , Quantum Symmetry, Differential Geometry of Finite Graphs, and Classification of Subfactors, Univ. of Tokyo Seminary Notes, 1991.

[31] S. Popa, Correspondences, preprint.

[32] Classification of actions of discrete amenable groups on amenable subfactors of type II, preprint.

[33] J.L. Sauvageot, Sur le produit tensoriel relatif d'espaces de hilbert, J. Operator Theory, 9 (1983), 237-252.

[34] E. Verlinde, Fusion rules and modular transformation in $2 D$ conformal field theory, Nucl. Phys., 300(B) (1988), 360-376.

[35] M. Wakui, Fusion algebras for orbifold models, preprint.

[36] S. Yamagami, A note on Ocneanu's approach to Jones' index theory, Internat. J. Math., 4 (1993), 859-871.

[37] , Modular theory in bimodules, J. Funct. Anal., 125 (1994), 327-357.

[38] Vector bundles and bimodules, in "Quantum and Non-commutative Analysis", Kluwer Academic, (1993), 321-329.

[39] , Frobenius reciprocity in tensor categories, preprint.

[40] T. Yoshida, On G-functors Part I: Transfer theorems for cohomological G-functors Hokkaido Math. J., 9 (1980), 222-257.

[41] , On G-functors Part II: Hecke operators and G-functors, J. Math. Soc. Japan, 35 (1983), 179-190.

Received September 10, 1994.

KYUSHU UNIVERSITY

Ropponmatsu, FuKuOKa 810, JAPAN

KYUSHU UNIVERSITY

HAKOZAKI, FUKUOKA 812 , JAPAN

AND

TOHOKU UNIVERSITY

SENDAI 980, JAPAN 